\title{
Satellite-Scale Snow Water Equivalent Assimilation into a High-Resolution Land Surface Model
}

\author{
GABRiËLle J. M. De LANNOY \\ George Mason University and Center for Research on Environment and Water, Calverton, Maryland, and \\ Laboratory of Hydrology and Water Management, Ghent University, Ghent, Belgium \\ ROLF H. REICHLE \\ Global Modeling and Assimilation Office, NASA Goddard Space Flight Center, Greenbelt, Maryland \\ PAul R. Houser AND Kristi R. ARsenault \\ George Mason University and Center for Research on Environment and Water, Calverton, Maryland \\ Niko E. C. Verhoest and VALentijn R. N. Pauwels \\ Laboratory of Hydrology and Water Management, Ghent University, Ghent, Belgium
}

(Manuscript received 8 July 2009, in final form 8 October 2009)

\begin{abstract}
Four methods based on the ensemble Kalman filter (EnKF) are tested to assimilate coarse-scale $(25 \mathrm{~km})$ snow water equivalent (SWE) observations (typical of passive microwave satellite retrievals) into finescale $(1 \mathrm{~km})$ land model simulations. Synthetic coarse-scale observations are assimilated directly using an observation operator for mapping between the coarse and fine scales or, alternatively, after disaggregation (regridding) to the finescale model resolution prior to data assimilation. In either case, observations are assimilated either simultaneously or independently for each location. Results indicate that assimilating disaggregated finescale observations independently (method 1D-F1) is less efficient than assimilating a collection of neighboring disaggregated observations (method 3D-Fm). Direct assimilation of coarse-scale observations is superior to a priori disaggregation. Independent assimilation of individual coarse-scale observations (method 3D-C1) can bring the overall mean analyzed field close to the truth, but does not necessarily improve estimates of the finescale structure. There is a clear benefit to simultaneously assimilating multiple coarse-scale observations $(\operatorname{method} 3 \mathrm{D}-\mathrm{Cm})$ even as the entire domain is observed, indicating that underlying spatial error correlations can be exploited to improve SWE estimates. Method 3D-Cm avoids artificial transitions at the coarse observation pixel boundaries and can reduce the RMSE by $60 \%$ when compared to the open loop in this study.
\end{abstract}

\section{Introduction}

Land surface data assimilation is mainly focused on surface temperature, soil moisture, and snow. These variables interact with the atmosphere, which explains their direct impact on weather and climate predictions (Koster et al. 2004; Dirmeyer 2000). Land surface variables have a large spatial variability that cannot be captured by the

Corresponding author address: Gabriëlle J. M. De Lannoy, Laboratory of Hydrology and Water Management, Ghent University, Coupure links 653, B-9000 Ghent, Belgium.

E-mail: gabrielle.delannoy@ugent.be existing operational observing systems alone. Land surface models could help in either downscaling the available coarse satellite observations or interpolating the scattered point observations. Snow differs from the other land surface states in its discontinuous local presence or absence, its cumulative character and often long temporal autocorrelation length (Slater and Clark 2006).

For snow, a variety of observations can be assimilated. First, there are numerous point-scale snow water equivalent (SWE) or snow depth measurements. Examples are long-term records from the U.S. Department of Agriculture's (USDA) Natural Resources Conservation Service (NRCS) Snowpack Telemetry (SNOTEL) network 
of snow pillow sites, the National Weather Service (NWS) Cooperative (COOP) weather stations network, and short-term spatially dense ground measurements from intensive field campaigns like the Cold Land Processes Experiments (CLPX). Direct point snow data assimilation has been demonstrated by Huang and Cressie (1996), Slater and Clark (2006), and Liston and Hiemstra (2008). Point-scale data have been widely used as validation for satellite data assimilation.

Satellite observations of snow cover area or fraction and SWE-related quantities provide a second type of (partial) information about the snow state. As examples, the Advanced Very High Resolution Radiometer (AVHRR), Landsat Thematic Mapper (TM) and Moderate Resolution Imaging Spectroradiometer (MODIS) provide snow cover and albedo products at fine resolutions $(\leq 1 \mathrm{~km})$, but these visible or near-infrared data cannot be collected at night or in cloudy conditions. Passive microwave sensor data do not suffer from these shortcomings, but have a much coarser resolution and come with large errors (Kelly et al. 2003; Pulliainen et al. 1999). The Advanced Microwave Scanning Radiometer for Earth Observing System (AMSR-E), the Scanning Multichannel Microwave Radiometer (SMMR), and the Spatial Sensor Microwave Imager (SSM/I) provide SWE information at a $25-\mathrm{km}$ resolution. Use of remote sensing data to update the SWE has focused on direct assimilation of MODIS snow cover area data (Rodell and Houser 2004; Andreadis and Lettenmaier 2006; Zaitchik and Rodell 2009) or inverted (real or synthetic) SWE data from passive microwave sensors (Sun et al. 2004; Andreadis and Lettenmaier 2006; Dong et al. 2007).

Third, different approaches have been developed to merge complementary sources of remotely sensed snow data (Durand et al. 2008), to combine remote sensing products with ground measurements (Pulliainen 2006; Tait et al. 2000), and to assimilate a priori merged snow products into models (Liston et al. 1999). The merger of different observational sources at multiple scales can also be achieved within the data assimilation framework, for example through the direct assimilation of multichannel passive microwave brightness and near-infrared data (Durand and Margulis 2006, 2007, 2008).

With few exceptions, notably Durand and Margulis (2007, 2008), snow data assimilation efforts have been limited to $1 \mathrm{D}$ filtering (i.e., the observations and model units were at, or brought to, the scale of the model grid and assimilated independently for each grid cell). The integration of coarse-scale observations for land surface state estimation in a dynamical framework is often complex, because satellite data and computational model units (grid cells, catchments units, etc.) typically differ in scale of support. With more advanced assimilation and scaling techniques, however, there is the potential to extract information through downscaling satellite-scale observations into higher-resolution land model integrations.

Coarse observations can be regridded to the finer modeling units prior to data assimilation. Examples include retrieving SMMR SWE over catchment units (Dong et al. 2007), and reconstructing MODIS snow cover and AMSR-E SWE to match the modeling scale (Andreadis and Lettenmaier 2006). Additionally, many synthetic studies take this approach, such as Sun et al. (2004) for SWE assimilation. More sophisticated a priori disaggregation approaches based on statistical relationships have been used for soil moisture (Dubayah et al. 1997; Kumar 1999; Crow and Wood 2002; Parada and Liang 2003; Merlin et al. 2006). In any case, the disaggregated products could then be useful for dynamical assimilation into a finer-scale land model.

Alternatively, the scale discrepancy can be dealt with more effectively and dynamically within the data assimilation framework by using a scaling observation operator. The disaggregation of coarse-scale information is then based on spatial error correlations that are modeled within the assimilation system. For example in land surface data assimilation, Reichle et al. (2001), Caparrini et al. (2004), Durand and Margulis (2007), and Zaitchik et al. (2008) used an averaging of a number of finescale land model forecasts to generate observation predictions at the coarse observation scale, respectively, for assimilation of soil moisture, land surface temperature, SWE, and water storage.

In this paper, techniques for downscaling coarse-scale SWE observations to the underlying finescale model state variables within data assimilation is described and tested for the first time within the National Aeronautics and Space Administration (NASA) Land Information System (Kumar et al. 2006; Peters-Lidard et al. 2007; Kumar et al. 2008a,b), version 5.0 (LIS5.0). One of the challenges in assimilating coarse observations over a large domain of finescale model units is in the ensemble estimation of the forecast error covariance matrix, where there is often a problem with spurious long-range correlations. Therefore, ensemble filter techniques mostly include some type of localization (Keppenne and Rienecker 2002; Ott et al. 2004; Hunt et al. 2006; Reichle and Koster 2003), which will be discussed in this paper as well. As an alternative, multiscale Kalman filters could provide a way to replace the forecast error covariance matrix with a multiscale tree (Zhou et al. 2008; Parada and Liang 2004, 2008; Pan et al. 2009).

A number of ensemble Kalman filter (EnKF) approaches are explored that combine finescale land surface model simulations and synthetic satellite-scale observations. The focus will be on (i) the difference 
between 1D (point) and 3D (spatial) filtering and (ii) the study of different 3D filter approaches in view of subpixel snow variability estimation. Reichle and Koster (2003) demonstrated that the 3D EnKF was superior to the 1D EnKF in a synthetical soil moisture estimation study. In that study, observations and modeling units corresponded in scale of support (catchments), and the advantage in the 3D filtering was primarily in datasparse regions, because of the horizontal information propagation through the spatial forecast error structure (Hamill and Snyder 2000). This advantage of 3D filtering is also illustrated by Houser et al. (1998) and De Lannoy et al. (2009). In the synthetic experiment discussed here, however, observations are available for the entire domain and the focus is on the spatial disaggregation of coarse-scale observational information typical of passive microwave satellite retrievals.

\section{Experiment setup}

Different filters are tested in an identical twin experiment. Snowpack evolution is simulated at the fine scale $(1 \mathrm{~km})$ as a reference "truth" for validation. A second integration with degraded forcing data mimics forecast modeling errors. Synthetic observations are generated from the truth integration at the coarse satellite scale $(25 \mathrm{~km})$ by averaging the finescale snowpack and adding observation error. The coarse observation assimilation into the finer-scale degraded model simulations is then validated against the finescale truth.

The study domain includes the North Park area and part of the Rabbit Ears area in Colorado, which have been intensively monitored during CLPX-I. The rectangular study area (left-bottom corner: $40.255^{\circ} \mathrm{N},-106.745^{\circ} \mathrm{W}$; top-right corner: $\left.40.995^{\circ} \mathrm{N},-105.755^{\circ} \mathrm{W}\right)$ includes $75 \times$ 100 finescale grid cells $\left(0.01^{\circ} \sim 1 \mathrm{~km}\right)$ covering a central valley surrounded by mountains (Fig. 1).

SWE is simulated with the land surface model of the National Centers for Environmental Prediction (NCEP)/ Oregon State University/Air Force/Hydrologic Research Laboratory (Noah version 2.7.1; Ek et al. 2003) at a $0.01^{\circ}$ $(\sim 1 \mathrm{~km})$ resolution for the winter of 2002-03, after a spinup starting in 2000. The NASA LIS5.0 (Kumar et al. 2008b) is used and adapted for this study. The forcings for the truth run are obtained from the North American Land Data Assimilation System [NLDAS, original resolution $1 / 8^{\circ}$, Cosgrove et al. (2003), mapped to $0.01^{\circ}$ through bilinear interpolation]. For all simulations a fixed temperature lapse rate of $-6.5 \mathrm{~K} \mathrm{~km}^{-1}$ is used to disaggregate temperature data (Mitchell et al. 2004). The LSM parameters include a MODIS-based land mask and land-cover parameters $(1 \mathrm{~km})$, the U.S. Geological Survey's (USGS) GTOPO30 elevation map $\left(\sim 0.01^{\circ}\right)$ to
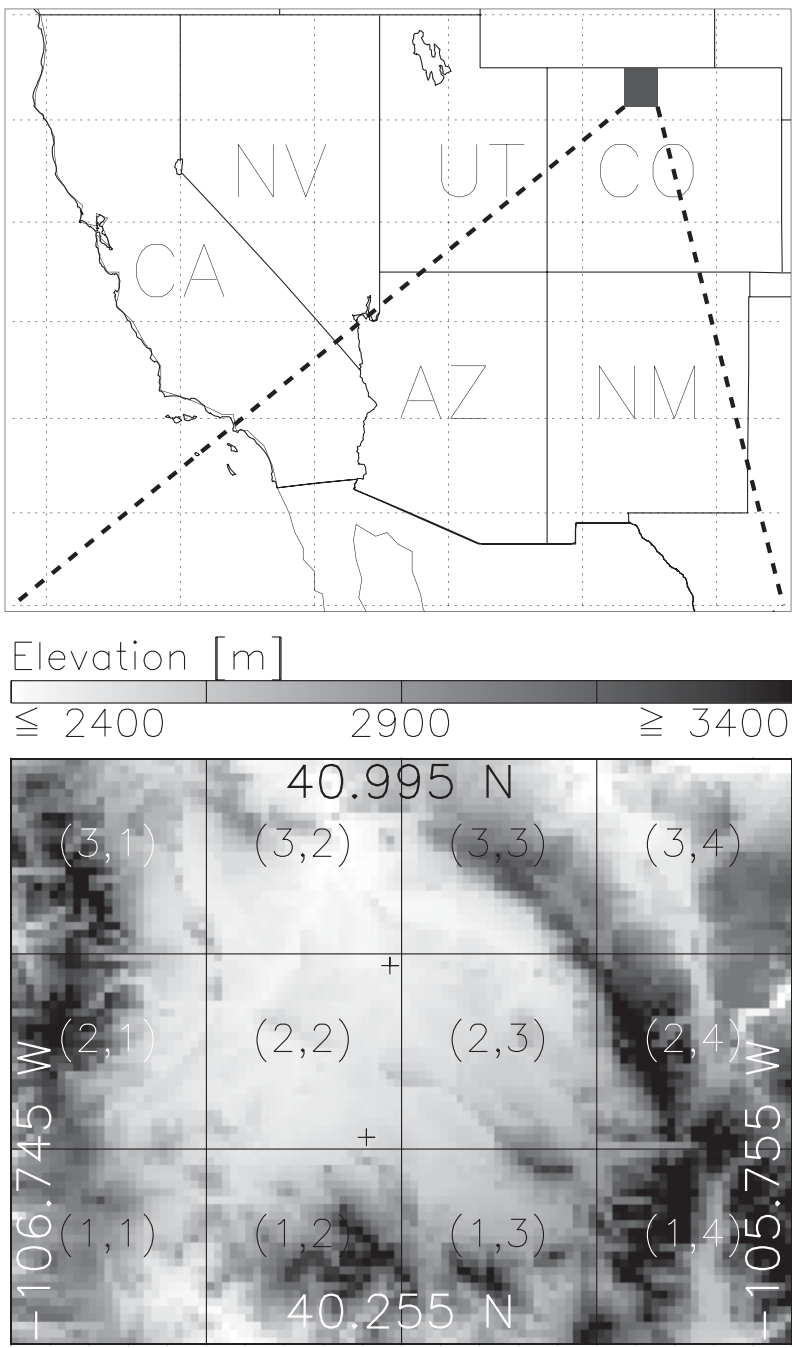

FIG. 1. (Top) location of the study area within the western United States and (bottom) the digital elevation model, overlaid by a $25 \mathrm{~km} \times 25 \mathrm{~km}$ resolution raster. The two plus symbols mark locations for which individual time series are plotted in Fig. 5, with the upper one at finescale row and column $(49,49)$ and the lower one at $(27,46)$.

correct NLDAS forcings at the 1-km scale, the Pennsylvania State University-U.S. Department of Agriculture (USDA) State Soil Geographic Database (STATSGO, $1 / 20^{\circ}$ ), a snow-free albedo product and a Noah-specific maximum snow albedo product from NCEP $\left(1^{\circ}\right)$, National Oceanic and Atmospheric Administration (NOAA)/AVHRR-based greeness fraction $\left(0.144^{\circ}\right)$, and an NLDAS-based climatological bottom temperature $\left(1 / 8^{\circ}\right)$. The default Noah soil, vegetation, and general parameter tables are used.

Synthetic SWE observations that mimic AMSR-E SWE retrievals are derived by aggregating the $1-\mathrm{km}$ reference truth SWE simulation into 12 coarse-scale $(25 \mathrm{~km} \times 25 \mathrm{~km})$ grid cells (3 rows, 4 columns, see Fig. 1 ). 
Random noise is added to mimic observation errors and drawn from a normal distribution with a SWE-dependent standard deviation. Specifically, we use $\sigma_{\text {obs }}=5+$ $0.095 \mathrm{SWE}(\mathrm{mm})$. The perturbation standard deviation is minimally $5 \mathrm{~mm}$, when no SWE is observed, and linearly increases to $100 \mathrm{~mm}$ for $1000-\mathrm{mm}$ SWE. The average observation error magnitude is in the range reported for real passive microwave data (i.e., $5-45 \mathrm{~mm}$ in nonforested areas), which increased by $5-10 \mathrm{~mm}$ over forests (Pulliainen et al. 1999; Derksen et al. 2003). Obviously, the assumed error function is only a crude approximation of actual errors. In practice, the type of snow emission model (Kelly et al. 2003; Pulliainen et al. 1999), local conditions and a number of physical details (including grain size, presence of liquid water, and vegetation; Foster et al. 2005; Dong et al. 2005) will strongly influence the observation error in satellite-based passive microwave SWE products. Furthermore, the AMSR-E products are known to have limited sensitivity to SWE for both very thin and deep snowpacks (Dong et al. 2005). Nevertheless, we keep the whole range of synthetic observations, to illustrate the potential of the different assimilation techniques.

A second set of Noah model forecasts (open loop, degraded model simulations) are generated using forcings from the Global Data Assimilation System (GDAS), which is coarser than NLDAS and originally at a Gaussian grid (T170 $\sim 0.7^{\circ}$ until 29 October 2002, T254 $\sim 0.5^{\circ}$ until 31 May 2005). The forcings are of major importance for the correct characterization of the SWE evolution (Slater et al. 2001; Mote et al. 2003), and their errors (Goodison 1978) will probably contribute most to SWE forecast errors in real experiments. Because the GDAS forcings result in heavily underestimated SWE simulations over the selected study domain, the precipitation is multiplied by a factor of 3 to correct for the otherwise large forecast bias during the assimilation. This correction factor was determined through trial and error. More sophisticated bias correction methods such as matching of the cumulative distribution functions (Reichle and Koster 2004) or dynamic bias estimation (De Lannoy et al. 2007) could be used, but are beyond the scope of this paper. The precipitation, air temperature, shortwave and longwave radiation, as well as the state variables are perturbed to generate ensembles for the assimilation, as will be discussed below.

The satellite-scale $(25 \mathrm{~km})$ synthetic observations are assimilated into the finescale $(1 \mathrm{~km})$ degraded model simulations twice per month (every 15 th and 30/28th of the month) from 30 September 2002 to 30 June 2003 (winter 2002-03). To mimic the AMSR-E overpasses, the assimilation time is set to 0800 UTC (0100 a.m. local time). The night overpass is selected (Durand and
Margulis 2007), because midday SWE retrievals are typically affected by liquid (melted) water in the snowpack.

This synthetic experiment circumvents major issues in real snow studies. As mentioned above, AMSR-E SWE observations are known to saturate for deep snowpacks. Furthermore, the snow simulations in our experiment have, by construction, the same climatology as the synthetic truth, which may well be different from the actual climatology (no ground truth validation was performed). The representation of snow physics is often too simple for complex terrains and snow forecast bias can be expected. Finally, point-scale SWE measurements are also subject to significant errors and their use as reference data (truth) to validate finescale model forecast or assimilation experiments is problematic.

\section{Data assimilation}

\section{a. Ensemble Kalman filter}

The EnKF is a Monte Carlo variant of the Kalman filter (Evensen 2003). The idea behind the EnKF is that a small ensemble of model trajectories captures the relevant parts of the forecast error structure. Each member of the ensemble experiences perturbed instances of the observed forcing fields (representing errors in the forcing data) and is also subject to randomly generated noise that is added to the model parameters and prognostic variables (representing errors in model physics and parameters). The error covariance matrices that are required for the filter update can then be diagnosed from the spread of the ensemble at the update time. The EnKF is flexible in its treatment of errors in model dynamics and parameters. It is also very suitable for modestly nonlinear problems and has become a popular choice for land data assimilation (Andreadis and Lettenmaier 2006; Durand and Margulis 2008; Kumar et al. 2008b; Pan and Wood 2006; Pauwels and De Lannoy 2006; Reichle et al. 2002a,b; Zhou et al. 2008).

The EnKF works sequentially by performing in turn a model forecast and a filter update. To reflect the uncertainty in the state forecasts, perturbations of the model forcings and the initial state estimate are applied at each time step to generate $N$ ensemble forecasts $\hat{\mathbf{x}}_{i}^{j-}(j=1, \ldots, N)$ with the model f:

$$
\hat{\mathbf{x}}_{i}^{j-}=\mathbf{f}\left(\hat{\mathbf{x}}_{i-1}^{j+}, \mathbf{u}_{i}, \mathbf{w}_{i-1}^{j}\right),
$$

where $i$ denotes time, $\hat{\mathbf{x}}_{i-1}^{j+}$ is the analysis from the previous time step (see below), $\mathbf{u}_{i}$ represents the forcings, and $\mathbf{w}_{i-1}^{j}$ denotes the model error or perturbations to the $j$ th ensemble member. The model forecast $\hat{\mathbf{x}}_{i}^{-}$is given by the ensemble mean. As a convention, we use bold uppercase symbols to refer to two-dimensional matrices, 
bold lowercase symbols for one-dimensional vectors, and nonbold italic symbols for scalars.

When observations $\mathbf{y}_{i}$ are available at time $i$, each ensemble member $j$ is updated individually to obtain the a posteriori state estimate (or analysis):

$$
\hat{\mathbf{x}}_{i}^{j+}=\hat{\mathbf{x}}_{i}^{j-}+\mathbf{K}_{i}\left[\mathbf{y}_{i}^{j}-\mathbf{h}_{i}\left(\hat{\mathbf{x}}_{i}^{j-}\right)\right]
$$

where $\mathbf{y}_{i}^{j}$ is a suitably perturbed observation vector (Burgers et al. 1998). The analysis $\hat{\mathbf{x}}_{i}^{+}$is again given by the ensemble mean. The function $\mathbf{h}_{i}$ is the observation operator that maps the state to the observation space, and $\mathbf{h}_{i}\left(\hat{\mathbf{x}}_{i}^{j-}\right)$ denotes the model's observation prediction for ensemble member $j$. The Kalman gain $\mathbf{K}_{i}$ is identical for all ensemble members and determined by the (sample) error covariance $\operatorname{Cov}\left[\hat{\mathbf{x}}_{i}^{-}, \mathbf{h}_{i}\left(\hat{\mathbf{x}}_{i}^{-}\right)\right]$between the forecast and the observation predictions, the (sample) error covariance $\operatorname{Cov}\left[\mathbf{h}_{i}\left(\hat{\mathbf{x}}_{i}^{-}\right), \mathbf{h}_{i}\left(\hat{\mathbf{x}}_{i}^{-}\right)\right]$of the observation predictions, and the observation error covariance $\mathbf{R}_{i}$ :

$\mathbf{K}=\operatorname{Cov}\left[\hat{\mathbf{x}}_{i}^{-}, \mathbf{h}_{i}\left(\hat{\mathbf{x}}_{i}^{-}\right)\right]\left[\operatorname{Cov}\left[\mathbf{h}_{i}\left(\hat{\mathbf{x}}_{i}^{-}\right), \mathbf{h}_{i}\left(\hat{\mathbf{x}}_{i}^{-}\right)\right]+\mathbf{R}_{i}\right]^{-1}$.

For a linear (or linearized) observation operator $\mathbf{H}_{i}$ [i.e., $\left.\mathbf{h}_{i}\left(\hat{\mathbf{x}}_{i}^{-}\right) \approx \mathbf{H}_{i} \hat{\mathbf{x}}_{i}^{-}\right]$, the sample error covariances can be written as

$$
\begin{aligned}
\operatorname{Cov}\left[\hat{\mathbf{x}}_{i}^{-}, \mathbf{h}_{i}\left(\hat{\mathbf{x}}_{i}^{-}\right)\right] & =\mathbf{P}_{i}^{-} \mathbf{H}_{i}^{\mathrm{T}} \text { and } \\
\operatorname{Cov}\left[\mathbf{h}_{i}\left(\hat{\mathbf{x}}_{i}^{-}\right), \mathbf{h}_{i}\left(\hat{\mathbf{x}}_{i}^{-}\right)\right] & =\mathbf{H}_{i} \mathbf{P}_{i}^{-} \mathbf{H}_{i}^{\mathrm{T}},
\end{aligned}
$$

where $\mathbf{P}_{i}^{-}$is the forecast error covariance and superscript $\mathrm{T}$ denotes the matrix transpose.

In this paper we use the EnKF modules of the NASA Global Modeling and Assimilation Office (GMAO; Reichle et al. 2009) within LIS5.0 (after modifying the LIS5.0 implementation to enable the GMAO EnKF capability for horizontal error correlations, covariance localization, and distributed filtering). In each finescale $(1 \mathrm{~km})$ grid cell, the Noah model simulates a single snow layer with two prognostic variables: one for total column snow water equivalent (swe) and one for snow depth (snd). The EnKF is used to update these two model prognostic variables at the $1-\mathrm{km}$ scale. Dropping the time index $i$, Eqs. (2) and (3) can then be rewritten for a given finescale grid cell $k$ as

$$
\begin{aligned}
\left(\begin{array}{c}
\operatorname{swe}_{k}^{j+} \\
\operatorname{snd}_{k}^{j+}
\end{array}\right) & =\left(\begin{array}{c}
\operatorname{swe}_{k}^{j-} \\
\operatorname{snd}_{k}^{j-}
\end{array}\right)+\mathbf{K}\left(\mathbf{y}^{j}-\hat{\mathbf{y}}^{j-}\right), \\
\mathbf{K} & =\operatorname{Cov}\left[\left(\begin{array}{c}
\operatorname{swe}_{k}^{-} \\
\operatorname{snd}_{k}^{-}
\end{array}\right), \hat{\mathbf{y}}^{-}\right]\left[\operatorname{Cov}\left(\hat{\mathbf{y}}^{-}, \hat{\mathbf{y}}^{-}\right)+\mathbf{R}\right]^{-1},
\end{aligned}
$$

with $\mathbf{y}^{j}$ denoting the perturbed SWE observations that are used to update $\operatorname{swe}_{k}^{j-}$ and $\operatorname{snd}_{k}^{j-}$, where $\hat{\mathbf{y}}^{j-} \equiv \mathbf{h}\left(\hat{\mathbf{x}}^{j-}\right)$ is shorthand for the corresponding model predictions of these observations, and where $\mathbf{R}$ denotes the corresponding observation error covariance.

The first term in the expression for the Kalman gain includes the error correlation between forecasted snow depth $\left(\mathrm{snd}^{-}\right)$and $\operatorname{SWE}\left(\hat{\mathbf{y}}^{-}\right)$. This correlation information is the basis for updating snow depth ( snd $\left.^{-}\right)$in response to SWE observations (y). If $m$ observations are used to update SWE and snow depth for a given finescale grid cell, the Kalman gain $\mathbf{K}$ in Eq. (5) is a $2 \times m$ matrix, and the observations $\mathbf{y}$ and observation predictions $\hat{\mathbf{y}}^{-}$are column vectors with $m$ elements. In sections $3 c$ and $3 d$, we will specify how exactly the coarse-scale SWE observations are used (to update the finescale model prognostic variables swe ${ }_{k}^{j-}$ and $\mathrm{snd}_{k}^{j-}$ ) by relating $\mathbf{y}^{j}$ and $\hat{\mathbf{y}}^{j-}$ to observation and model variables. In any case, successful downscaling of coarse-scale observational information relies on ancillary information at the smaller scale (e.g., from micrometeorological or terrain information) and on horizontal error correlations that enable horizontally distributed updates.

\section{b. Ensemble generation}

The key to a successful merger of observational information into the land model is the Kalman gain $\mathbf{K}$. The elements of $\mathbf{K}$ are determined by the characteristics of the model forecast and observation errors, which are modeled by generating ensembles of perturbation fields with specified variances as well as spatial, temporal, and cross-correlation parameters. Random perturbation fields (i.e., normally distributed, zero mean, spatially correlated, temporally uncorrelated) are added hourly to the longwave radiation ( $\mathrm{LW}$, standard deviation, $\mathrm{stdv}=10 \mathrm{~W} \mathrm{~m}^{-2}$ ) and near-surface air temperature $(\mathrm{AT}, \mathrm{stdv}=1 \mathrm{~K})$ forcings, as well as to the forecasted SWE $(\mathrm{stdv}=2.5 \mathrm{~mm})$ and snow depth $(\mathrm{stdv}=0.01 \mathrm{~m})$. The precipitation $(P)$ and shortwave radiation (SW) are perturbed through multiplication with a random log-normal distributed field with $s t d v=0.2$ and $s t d v=0.5$, respectively, for the standard deviation of the multiplication factor. Cross correlation between the forcing perturbations is included (LW-SW: -0.3, SW-P: -0.1, SW-AT: 0.3, LW-P: 0.5, LW-AT: 0.6 , P-AT: -0.1 , assuring both positive definiteness and some balancing of forcing fields; Reichle et al. 2007), as well as cross correlation between swe ${ }^{-}$and snd $^{-}$forecast state (i.e., 0.9) perturbations. The latter are added after the state forecast simulation to account for model structure and parameter errors. The magnitude of the perturbations is chosen in a realistic range and checked to allow near-optimal filter performance [see section $4 b(5)$ ].

Experiments are conducted with several different spatial correlation lengths (1_corr) of the perturbation fields 
for the above-mentioned variables. Values of 1 corr range between approximately 5 and $100 \mathrm{~km}$ (i.e., $0.05^{\circ}$, $0.1^{\circ}, 0.2^{\circ}, 0.3^{\circ}, 0.5^{\circ}, 0.7^{\circ}$, and $1^{\circ}$ ) and are always chosen to be identical for all different perturbation fields in a given experiment for simplicity and because the estimates for error correlation scales are in any case very uncertain. When the spatial correlations are not included in the Kalman gain calculation, then the choice of 1_corr does not matter and the spatial correlations are effectively forced to zero in the Kalman gain calculation. The number of ensembles is set to $N=12$. A limited sensitivity test on the ensemble size did not show any significant qualitative difference in the overall result trends.

\section{c. Observation preprocessing: A priori observation disaggregation (1D-F1 and $3 D-F m)$}

Perhaps the simplest approach for the assimilation of coarse-scale observations into finer-scale model simulations is to create a surrogate observational grid with the same resolution as the finescale model grid. Here, the simplest possible disaggregation operation is used by assigning to each finescale $(1 \mathrm{~km})$ grid cell the value of the observation in the coarse $(25 \mathrm{~km})$ grid cell that contains the finescale grid cell. Alternatively, complementary data sources could be used to a priori disaggregate coarse observations (e.g., coarse-scale AMSR-E SWE and finescale MODIS snow cover; Liang et al. 2008).

An easy assimilation approach is then to apply the EnKF independently at each finescale grid cell $k$, using a single disaggregated and collocated observation swe ${ }_{k}^{\text {obs }}$ as illustrated in Fig. 2. In this approach, spatial forecast error correlations are entirely disregarded. This filter is referred to as 1D-F1, a one-dimensional (1D) filter that uses exactly one finescale observation (F1) for the analysis increment computation at a given finescale grid cell. Formally, we compute the analysis increments $\mathbf{K}\left(\mathbf{y}^{j}-\hat{\mathbf{y}}^{j-}\right)$ for the entire domain by looping through all finescale grid cells $k$ and using (for each $k$ )

$$
\begin{aligned}
\mathbf{y}^{j} & =\operatorname{swe}_{k}^{\mathrm{obs}, j} \\
\hat{\mathbf{y}}^{j-} & =\operatorname{swe}_{k}^{j-}
\end{aligned}
$$

as inputs to Eq. (5).

A straightforward extension of the 1D-F1 approach is to use several $(\mathrm{m})$ neighboring observations (after disaggregation to the finescale) in the analysis increment computation for a given finescale grid cell $k$ (Fig. 2). This approach limits the edge effect at the transition line between neighboring coarse observation pixels through the consideration of horizontal error correlations in the Kalman gain computation. We refer to this approach

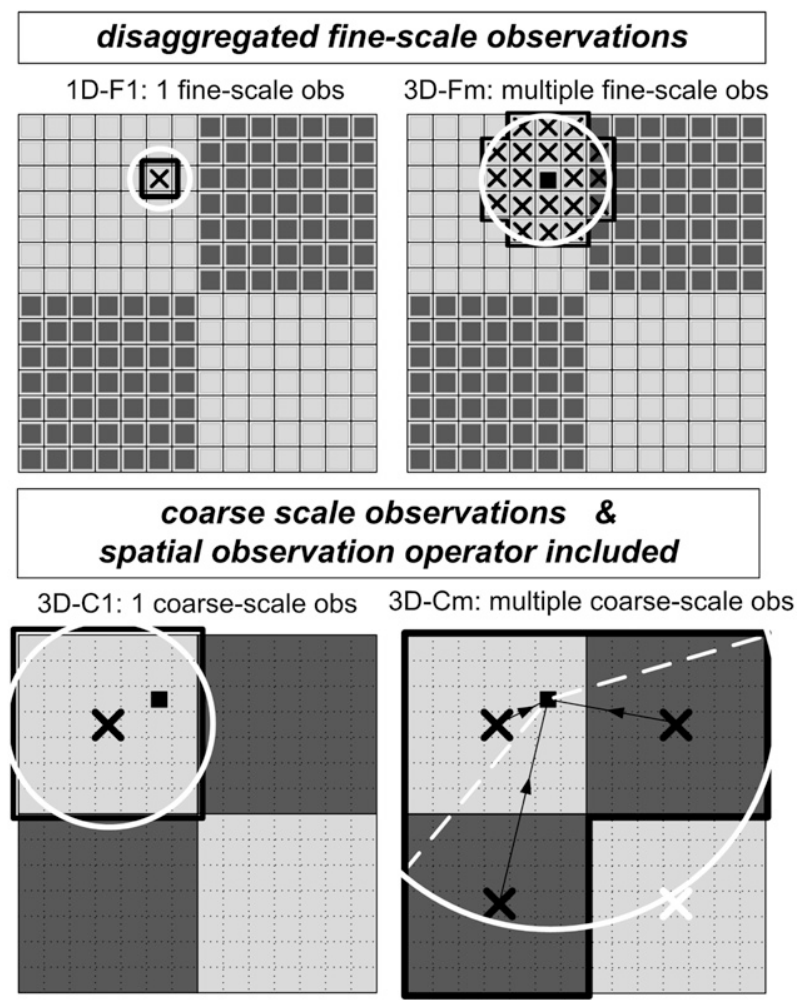

FIG. 2. Schematic of EnKF approaches illustrated for four coarse-scale pixels (gray shading), each containing $7 \times 7$ finescale pixels. For the state update of a given finescale pixel (black filled square), we use observations within an influence area (white circle) and a corresponding set of finescale model forecasts (thick black line). For 1D-F1 and 3D-Fm finescale (disaggregated) observations are used (small crosses). For 3D-C1 and 3D-Cm, coarse-scale observations are used (center locations are indicated by larger crosses). In the $3 \mathrm{D}$-Cm example, the observation at the white cross location is outside the influence area and not used to update the marked finescale state variable.

as 3D-Fm, because horizontal error correlations are taken into account in a "three-dimensional" (3D) filtering approach (following the convention of Reichle and Koster 2003, even though technically the land model has only one snow layer and our case is strictly speaking "2D" assimilation) and because $m$ (disaggregated) finescale (Fm) observations are used to update a given finescale grid cell. Formally, we compute the analysis increments for the entire domain by looping through all finescale grid cells $k$ and using (for each $k$ )

$$
\mathbf{y}^{j}=\left(\begin{array}{c}
\mathrm{swe}_{k_{1}}^{\mathrm{obs}, j} \\
\mathrm{swe}_{k_{2}}^{\mathrm{obs}, j} \\
\cdots \\
\mathrm{swe}_{k_{m}}^{\mathrm{obs}, j}
\end{array}\right)
$$




$$
\hat{\mathbf{y}}^{j-}=\left(\begin{array}{c}
\mathrm{swe}_{k_{1}}^{j-} \\
\mathrm{swe}_{k_{2}}^{j-} \\
\cdots \\
\operatorname{swe}_{k_{m}}^{j-}
\end{array}\right)
$$

as inputs to Eq. (5), where $k_{1}, k_{2}, \ldots, k_{m}$ indicate the $m$ (finescale) observations and predictions that are included in the update of finescale grid cell $k$. The choice of the observation selection area (influence radius around the analysis grid cell $k$ ) determines the observation dimension $m$. Because a larger influence radius also means a more complicated inversion for the Kalman gain calculation, we limit the influence radius to $10 \mathrm{~km}$ when $1-\mathrm{km}$ resolution observations are used (maximum $m=$ $317, m$ is smaller near the domain boundaries). The observation error covariance matrix $\mathbf{R}_{i}$ is assumed diagonal. Obviously, as the influence radius approaches zero, the 3D-Fm approach reduces to the 1D-F1 method.

In our synthetic experiment, the dynamic observation error variance is known for the coarse-scale observations, but not necessarily for the disaggregated observations. The observation error includes both the instrument error and the representativeness error of an observation operator. While the first error term is typically assumed to be white Gaussian, the second one can affect the actual observation error magnitude and correlations for the disaggregated observations. For simplicity, we do not consider spatially correlated observation errors and the same observation error magnitude is used for both fineand coarse-scale observation assimilation.

\section{d. Observation operator: Forecast upscaling $(3 D-C 1$ and $3 D-C m)$}

It is more appealing to avoid the observation disaggregation prior to assimilation and to perform the downscaling within the filtering algorithm instead (Reichle et al. 2001; Durand and Margulis 2007; Zaitchik et al. 2008). Two disparate grids are then present in the multiscale filtering algorithm: the coarse observation grid and the finescale simulation grid, indicated by Greek and Roman letters, respectively. For example, a coarse-scale observation is denoted with $\mathrm{swe}_{\kappa}^{\text {obs }}$.

A first option is to use only the single coarse-scale observation $\mathrm{swe}_{\kappa}^{\mathrm{obs}}$ that covers the finescale grid cell $k$ to update $\mathrm{swe}_{k}^{j-}$ and $\mathrm{snd}_{k}^{j-}$ with Eq. (5) as illustrated in Fig. 2. In this case, the coarse-scale observation predictions are computed as the appropriate average over the corresponding finescale model predictions. Formally, we have

$$
\mathbf{y}^{j}=\mathrm{swe}_{\kappa}^{\mathrm{obs}, j}
$$

$$
\hat{\mathbf{y}}^{j-}=\frac{1}{625} \sum_{l=1}^{625} \mathrm{swe}_{k_{l}}^{j-},
$$

where $k_{l}, l=1, \ldots, 625$ indexes the $25 \times 25$ finescale $(1 \mathrm{~km})$ grid cells contained within the coarse-scale $(25 \mathrm{~km})$ grid cell $\kappa$. This approach is referred to as the 3D-C1 EnKF here, because it uses one coarse-scale observation pixel to update each finescale state. The error correlation between the finescale model state (to be updated) and the coarse-scale observation prediction [first term of the Kalman gain in Eq. (5)] then downscales the observational information into finescale updates.

The 3D-C1 technique can be expanded to include surrounding coarse observations in the analysis, thereby using spatial forecast error correlations that overarch the boundaries between the coarse observation pixel areas. Now, multiple $(\mathrm{m})$ coarse-scale observations are used. Formally, Eq. (5) will be used with

$$
\begin{gathered}
\mathbf{y}^{j}=\left(\begin{array}{c}
\mathrm{swe}_{\kappa_{1}}^{\mathrm{obs}, j} \\
\mathrm{swe}_{\kappa_{2}}^{\mathrm{obs}, j} \\
\ldots \\
\mathrm{swe}_{\kappa_{m}}^{\mathrm{obs}, j}
\end{array}\right), \\
\hat{\mathbf{y}}^{j-}=\left(\begin{array}{c}
\frac{1}{625} \sum_{l=1}^{625} \mathrm{swe}_{k_{l, 1}}^{j-} \\
\frac{1}{625} \sum_{l=1}^{625} \mathrm{swe}_{k_{l, 2}}^{j-} \\
\cdots \\
\frac{1}{625} \sum_{l=1}^{625} \mathrm{swe}_{k_{l, m}}^{j-}
\end{array}\right),
\end{gathered}
$$

where $\kappa_{m}, m=1,2, \ldots, 12$ denotes a coarse-scale grid cell in our study domain and $k_{l, m}, l=1,2, \ldots, 625$ indexes the finescale grid cells contained within $\kappa_{m}$. This technique is referred to as $3 \mathrm{D}-\mathrm{Cm}$, involving multiple coarse-scale observations and in a 3D filtering approach. As in Reichle and Koster (2003) we suppress spurious long-range correlations in the forecast ensemble through (element wise) Hadamard multiplication of the sample error covariance terms in Eq. (5) with a distancedependent and compactly supported function. Specifically, we use the fifth-order polynomial [Eq. (4.10) of Gaspari and Cohn (1999)] and set the compact support scale equal to 2.5 times the spatial correlation length 1_corr that is used for the ensemble spatial perturbation fields (i.e., $2.5^{\circ}, 1.75^{\circ}$, $1.25^{\circ}, 0.75^{\circ}, 0.5^{\circ}$, and $0.25^{\circ}$ ). Beyond $2.5 \times 1$ _corr (near the "range" of the correlation function), the correlation drops to insignificant values. In practice, the localization means 
that observations beyond a given distance from a specific finescale grid cell cannot affect the analysis at that grid cell.

Equation (5) is written for a single finescale grid cell $k$ but involves knowledge of a potentially large number of finescale model states for the observation prediction $\left(\hat{\mathbf{y}}^{j-}\right)$ computation. Furthermore, neighboring finescale grid cells may need the same innovation information for their updates. Updating the state by looping through each grid cell $k$ is thus not necessarily the most efficient algorithm. Here, we implemented the 3D-C1 and 3D-Cm update methods by looping through coarse-scale grid cells and simultaneously updating all 625 finescale grid cells within a given coarse grid cell.

\section{Results}

The assimilation results are validated against the reference truth at the fine scale. Unless otherwise specified, the results are analyzed for assimilation twice a month (19 events between 30 September 2002 and 30 June 2003) over the entire domain of 7500 finescale $1 \mathrm{~km}^{2}$ simulation grid cells (covered by 12 coarse-scale observation pixels).

\section{a. SWE open loop integration and coarse observations}

We first examine the synthetic coarse-scale observations and the ensemble mean open loop (Ens OL; no assimilation) integration. The top three rows of Fig. 3 show five snapshots of the SWE field for the truth integration, the observations, and the open loop integration, respectively. Figure 4 shows the root-mean-square error (RMSE) of SWE (in time and in space) along with a time series of the domain average SWE. By construction the domain average SWE for the observations very closely approximates the true domain average SWE. Note also that the open loop fields for the various choices of perturbation correlation lengths (1_corr) are nearly identical, thus we do not distinguish between them for plotting. The observations show a smaller RMSE than the open loop integration. Because the observations are based on the truth simulation, there is no bias in the observations. For the open loop integrations, by contrast, some local or temporal bias (in addition to random errors) cannot be avoided.

Table 1 summarizes the space-time RMSE over the 19 analysis time steps. The RMSE for the observations is about $44 \mathrm{~mm}$. As mentioned above, the ensemble mean open loop simulations for different 1_corr perform similarly, with an RMSE of about $77 \mathrm{~mm}$.

The difference between the truth and the open loop is caused by the difference in NLDAS and (scaled) GDAS forcings. Even though the GDAS forcings are at a very coarse resolution, the modeled SWE distribution is quite reasonable. This is because the temperature correction (lapse rate of $-6.5 \mathrm{~K} \mathrm{~km}^{-1}$ ) downscales the temperature and allows a finescale discrimination between snowfall and rainfall. Also, recall that a multiplication factor of 3 was used for the precipitation to bring the GDAS snowfall input to a more realistic level and obtain reasonable SWE amounts. The peak in the spatial RMSE for both the open loop estimates and observations occurs during the melt period. This is caused by an increasing patchiness and variability in the actual snow amounts (as evidenced in the last column of Fig. 3), which cannot be reflected in the coarse-scale observation and also not fully captured by the open loop integration. The open loop shows almost a month delay in melting off all snow during early spring (Fig. 4), because of an accumulated snowpack error.

Figure 5 shows a detail of the temporal SWE evolution for two individual finescale pixels. At the fine scale, the coarse observations can differ significantly from the truth [e.g., during the snow accumulation in pixel (49, 49)], because they do not represent the local fine scale SWE evolution. This finescale observation bias is important to keep in mind for 1D filtering with disaggregated observations. Note also that the SWE forecast uncertainty ( $\mathbf{P}_{i}^{-}$as measured by the ensemble spread; not shown) increases from zero when no snow is present to a maximum value during deep snowpacks, and then drops back to zero again after snowmelt.

\section{b. SWE analyses}

\section{1) SPACE-TIME-AVERAgEd Statistics}

For most filter scenarios the space-time average RMSE (Table 1) is lower than for either the open loop $(77 \mathrm{~mm})$ or the observations $(44 \mathrm{~mm})$. This indicates that both the spatial mean field and the finescale variability are improved and supports the premise that assimilation products are better than either forecasts or observations alone (Reichle and Koster 2005).

The 1D-F1 and 3D-Fm results show a similar RMSE (40-44 mm). Larger reductions in RMSE can be observed for the 3D-C1 and 3D-Cm with 1_corr $>20 \mathrm{~km}$. For just 19 assimilation events, the 3D-Cm approach achieves an RMSE decrease up to $60 \%$ when compared to the open loop and up to $25 \%$ when compared to the observations. For 3D-C1, the RMSE values are slightly higher (around $38 \mathrm{~mm}$ ) than for 3D-Cm (around $34 \mathrm{~mm}$ ) for 1_corr $>20 \mathrm{~km}$, but for shorter 1_corr the 3D-Cm shows less assimilation impact. With daily assimilation, the RMSE over the same 19 time steps could not be significantly reduced. In the following sections we analyze these findings in more detail. 
SWE $[\mathrm{mm}]$ \begin{tabular}{llllll}
\hline 2 & 62 & 122 & 182 & 242 & 2302
\end{tabular}
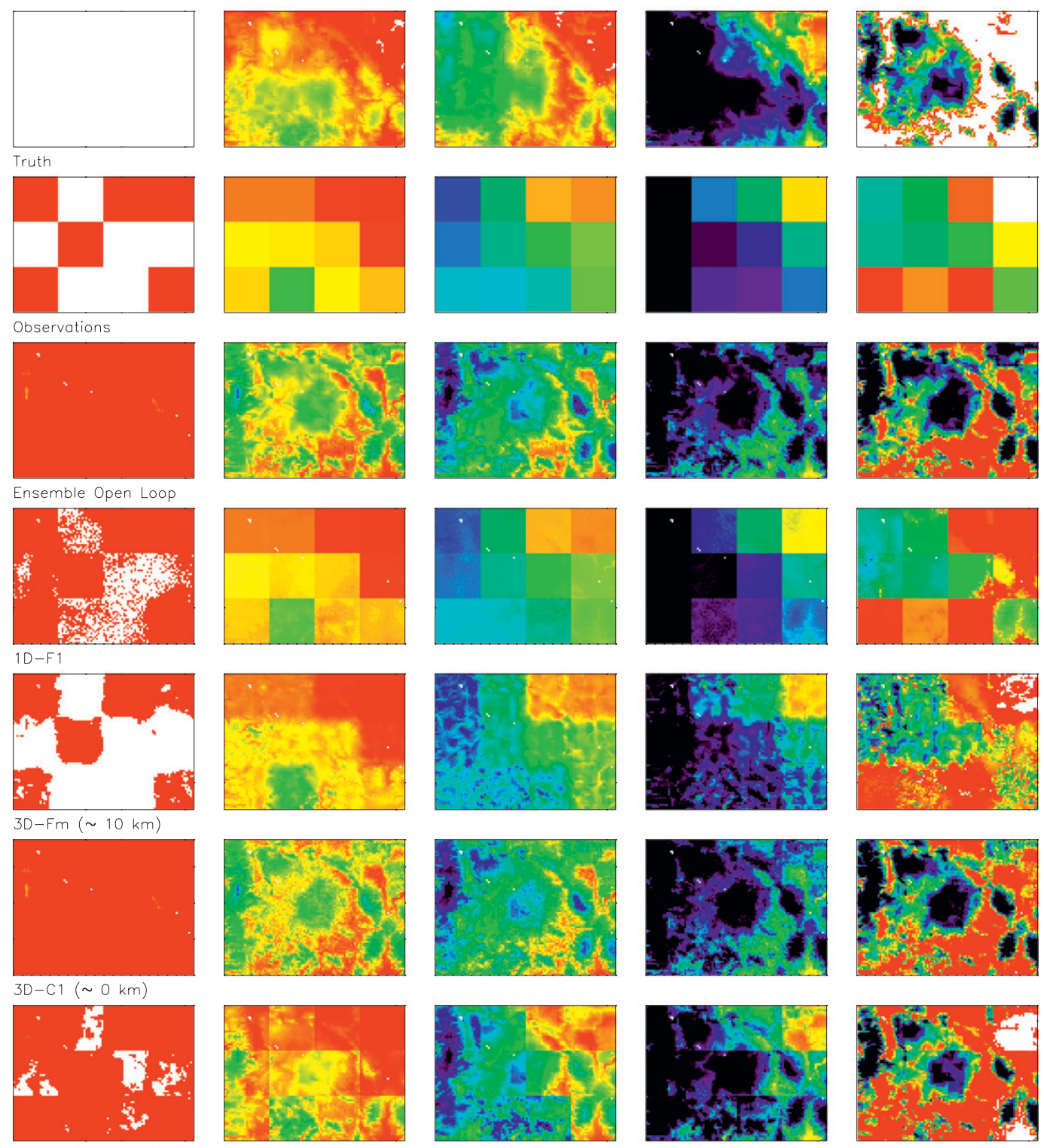

$3 \mathrm{D}-\mathrm{C} 1(\sim 30 \mathrm{~km})$
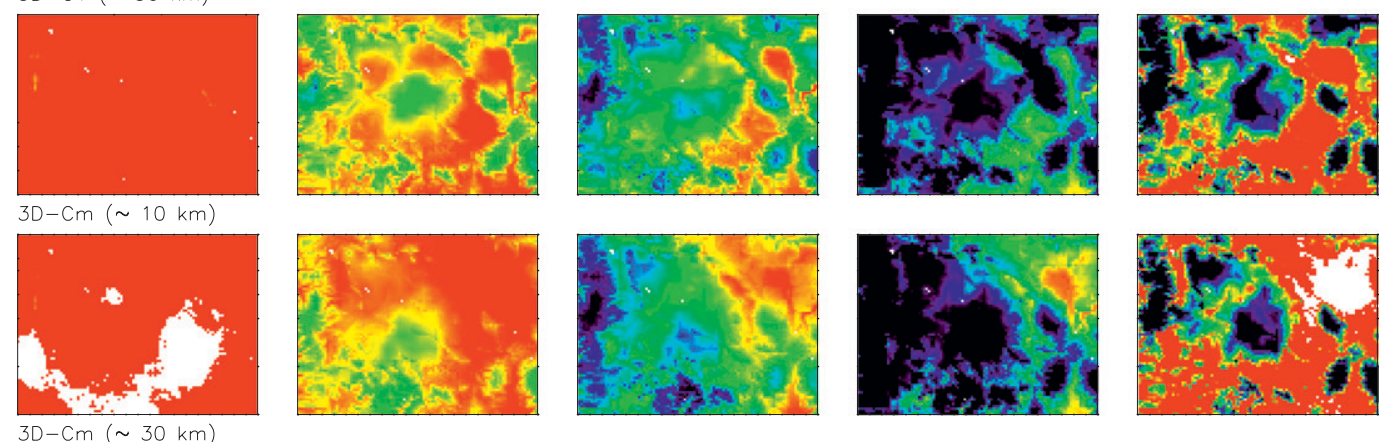

FIG. 3. (left to right) Snapshots of SWE fields for 15 Oct 2002, 30 Nov 2002, 15 Jan 2003, 28 Feb 2003, and 15 Apr 2003. (top to bottom) Truth, synthetic observations, ensemble mean open loop forecasts, and analyses obtained with several filter approaches. The spatial correlation length 1_corr in the forecast perturbations is indicated in parentheses. 
Temporal RMSE [mm]
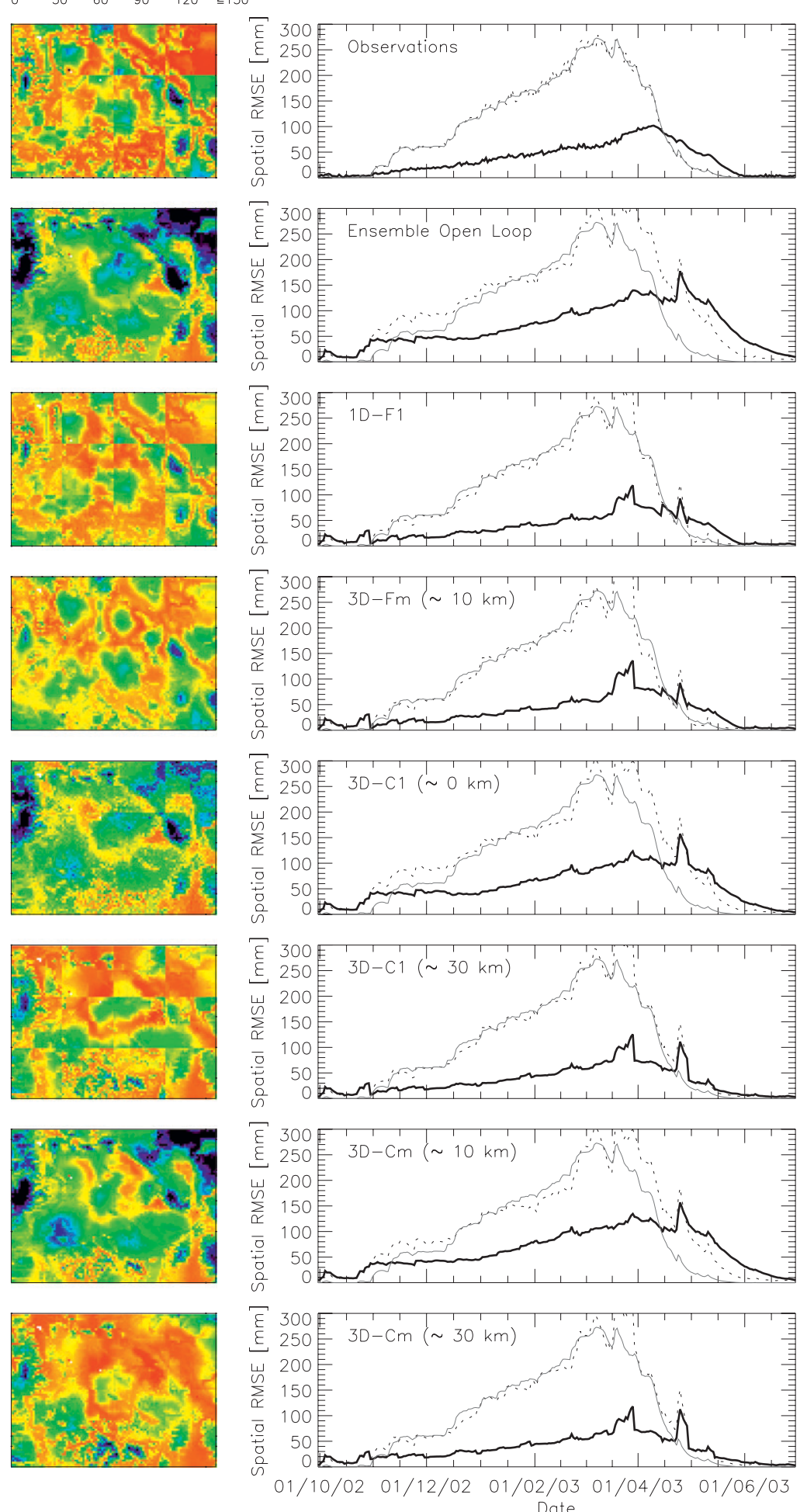

FIG. 4. (left) Temporal (colors) and (right) spatial RMSE (bold black line) of the finescale analysis SWE for the same assimilation scenarios as in Fig. 3. All analysis and forecast time steps at 0800 UTC in the period between 30 Sep 2002 and 30 Jun 2003 are included. The dashed black line represents the total domain averaged SWE. The solid gray line shows the true domain-averaged SWE. 
TABLE 1. RMSE of SWE (mm) over 19 update times and 7500 finescale locations, with differences between the truth and Obs, Ens OL, and different assimilation analyses. The quantity 1_corr is the spatial correlation length in the forecast perturbation fields.

\begin{tabular}{lcccccccc}
\hline \hline 1_corr & $0 \mathrm{~km}$ & $5 \mathrm{~km}$ & $10 \mathrm{~km}$ & $20 \mathrm{~km}$ & $30 \mathrm{~km}$ & $50 \mathrm{~km}$ & $70 \mathrm{~km}$ & $100 \mathrm{~km}$ \\
\hline Obs & 44.4 & N/A & N/A & N/A & N/A & N/A & N/A & N/A \\
Ens OL & 77.5 & 77.2 & 77.1 & 77.0 & 75.4 & 76.4 & 78.2 & 78.2 \\
1D-F1 & 40.9 & 41.0 & 41.0 & 40.9 & 40.8 & 40.8 & 40.2 & 40.2 \\
3D-Fm & N/A & 43.8 & 42.2 & N/A & N/A & N/A & N/A & N/A \\
3D-C1 & 61.7 & 53.1 & 42.0 & 39.0 & 37.1 & 38.0 & 39.2 & 37.7 \\
3D-Cm & N/A & 77.2 & 70.4 & 41.7 & 35.4 & 33.7 & 34.5 & 32.3 \\
\hline
\end{tabular}

\section{2) TEMPORAL AND SPATIAL SWE PATTERNS}

Figure 3 shows the spatial analysis SWE pattern for the different filter approaches. The time series of spatial RMSE for the assimilation analyses show a relatively smooth evolution (Fig. 4). This means that the updates are accepted without significant drift in the spatial mean and that the snowpack has a considerable memory. During early snow accumulation, none of the filters, except the 3D-Fm (see dip in the spatial RMSE evolution in Fig. 4 and spatial field in Fig. 3 on 15 October 2003), is able to remove the excessive snow to match the true absence of snow. A very thin layer is kept, which is generated through the ensemble perturbation. During this initial period with extremely low snow amounts the update is negligible, because the model spread is still negligible, while the observation uncertainty has a set minimum value of $5 \mathrm{~mm}$. During the ablation period, the open loop snowmelt delay can be largely reduced (Fig. 4) as a result of earlier snowpack corrections and the few instantaneous updates during the melt. The melt delay cannot be reduced for the filter scenarios relying excessively on the model predictions and hence resulting in analyses close to the open loop, like 3D-C1 with 1_corr = $0 \mathrm{~km}$ and 3D-Cm with very low 1_corr and a small localization scale. With a limited spatial error correlation, the error covariance between a single finescale grid state and the observation prediction (based on a number of finescale grid elements) is low and the Kalman gain is limited.

In the spatial dimension, a clear coarse block structure in the analyses is evident for the 1D-F1, 3D-Fm, and 3D-C1 filters (Fig. 3). The 1D-F1 strongly forces all finescale state values to the observed coarse-scale SWE. Toward the melt season, the forecasts have a reduced spread and the spatial structure of the model simulations becomes apparent. For the 3D-Fm with an influence radius of $10 \mathrm{~km}$, the coarse pixel boundaries are slightly smoothed, but they are still very obvious. By design, the $3 \mathrm{D}-\mathrm{C} 1$ will always show the coarse pixel boundaries, because the state variables within a coarse area are all updated using only the single overlying coarse observation.

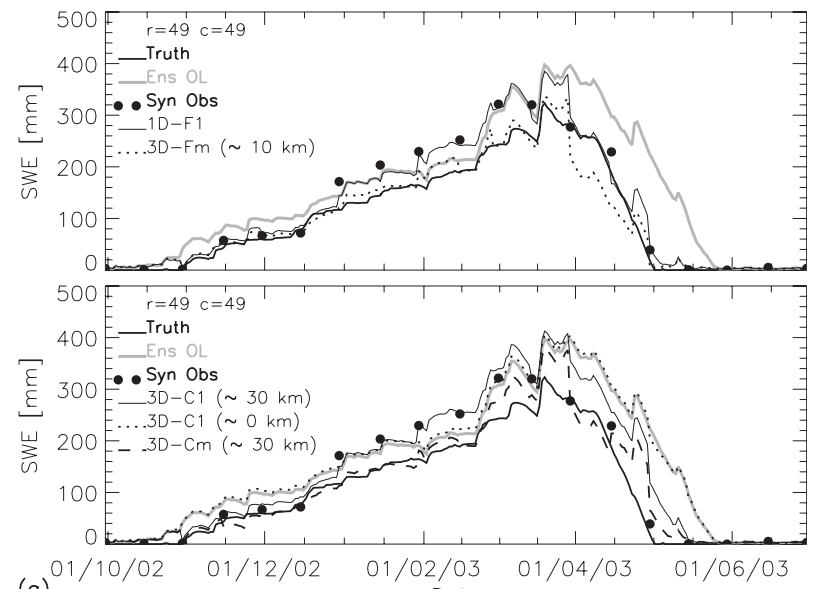

(a)

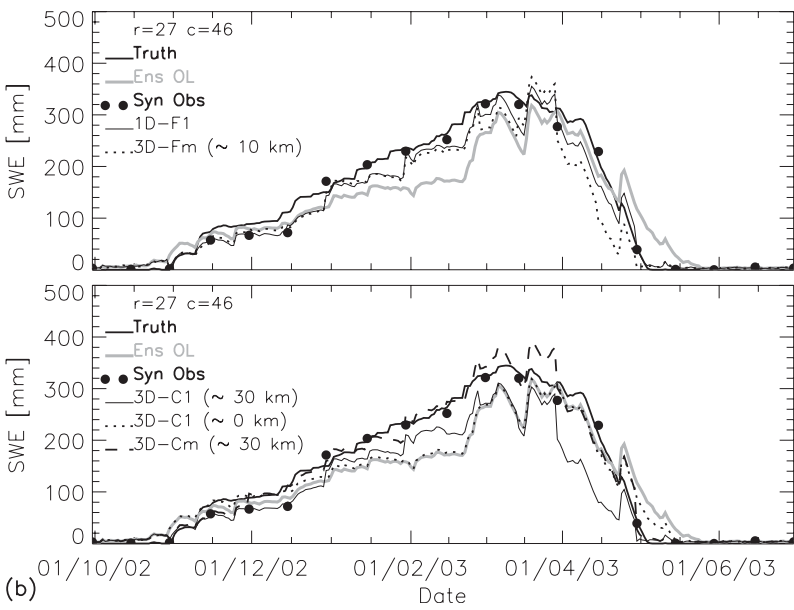

FIG. 5. Observed SWE (Syn Obs), Ens OL, and results for different assimilation algorithms at (a),(b) two finescale locations in the same coarse observation area (see Fig. 1). Only the assimilated observations are shown and they are identical for both finescale locations. Assimilation is performed twice each month.

However, when 1_corr $=0 \mathrm{~km}$, the boundaries are less noticeable, because of a negligible observation impact (see above). By construction, the 3D-Cm does not show coarse observation transitions in the analysis result, since it uses a collection of coarse observations centered on each individual analysis point.

The spatial pattern of the RMSE (Fig. 4) again shows the block patterns for some filters. Here, it is also clear that the spatial variability in the RMSE reduces with a longer 1_corr for 3D-C1 and 3D-Cm, and finescale locations with particular high RMSE values in the open loop integration are generally much improved through assimilation.

\section{3) SPATIAL FORECAST ERROR CORRELATION AND LOCALIZATION}

The results for the 3D-Cm and the 3D-C1 perform poorly when 1_corr $<20 \mathrm{~km}$ (Table 1). No drastic change 
in performance is found with 1_corr $>20-30 \mathrm{~km}$. This threshold distance is roughly equal to the dimension of the coarse-scale observation, and also approximates the mean of the observed precipitation error correlation length (as measured by analyzing the spatial correlation length in the NLDAS minus GDAS precipitation difference fields), although the latter was found to vary significantly in time (not shown).

For 3D-C1, updates near the borders in the coarse observation pixel may be limited, when 1_corr is smaller than the pixel dimension. In the border areas, the average distance to all other finescale pixels within the coarse observation area is longer than for center pixels, and the cross correlation with finescale variables at longer distances is smaller, especially when the distance reaches beyond 1_corr. This causes a limited covariance $\operatorname{Cov}\left[\hat{\mathbf{x}}_{i}^{-}, \mathbf{h}_{i}\left(\hat{\mathbf{x}}_{i}^{-}\right)\right]$between the forecast error in a single finegrid location and an observation prediction that is based on a number of finescale grid forecasts. The sample observation prediction error covariance $\operatorname{Cov}\left[\mathbf{h}_{i}\left(\hat{\mathbf{x}}_{i}^{-}\right), \mathbf{h}_{i}\left(\hat{\mathbf{x}}_{i}^{-}\right)\right]$ (which is independent of the finescale update location) is also smaller for shorter 1_corr. Therefore, a smaller 1_corr results in a smaller gain factor, mainly for the finescale analysis closer to the borders. For 1_corr $>20$ $30 \mathrm{~km}$, the forecast error correlations are near-uniformly high over each individual coarse satellite pixel area. Increasing the 1_corr beyond the coarse pixel dimension adds little to the covariances in the Kalman gain for each finescale update within the coarse pixel. This may explain why Durand and Margulis (2008) also found a maximum assimilation efficiency for an exponential variogram correlation length of $25 \mathrm{~km}$ when assimilating $25-\mathrm{km}$ resolution SWE-related observations, without significant degradation for longer 1_corr.

For the 3D-Cm approach, multiple coarse observations are included in each finescale update and a covariance localization scale equal to $2.5 \times 1$ _corr is applied to limit the impact of more distant observations. For small 1_corr (relative to the domain size), this avoids adverse effects caused by spurious long-range correlations. However, the smaller 1_corr and the localization limit the magnitude of the covariances in the calculation of the Kalman gain. This results in analyses close to the open loop simulation (cf. the spatial and temporal RMSE in Fig. 4 and see the analysis RMSE in Table 1 for 1_corr $=5$ and $10 \mathrm{~km}$ ). For longer 1_corr, the corresponding longer covariance localization scale only marginally reduces the sampled spatial correlations in this bounded study area. With longer 1_corr, the update is increased locally and spread to other locations as well (smoothing), because of larger forecast ensemblebased error covariances in the calculation of the Kalman gain.
In our study, a spatially isotropic error correlation and localization is imposed on the random fields, but the error field may not exactly represent the actual forecast error structure. Given the large elevation gradients and forecast errors introduced by different forcings, a complex error field can be expected. A good characterization of the error field and a region-dependent localization may further improve the results.

\section{4) Finescale SWE VARIABILITY}

Figure 6 shows scatterplots of the finescale assimilation estimates (forecasts and analyses) within a single coarse grid cell $(2,2)$ versus the true values just before and just after one particular assimilation update. The assimilated coarse-scale observation is also shown. These plots explain how each assimilation algorithm has a different effect on the spatial mean value and spread in the finescale SWE analyses. With the 1D-F1 filter, all finescale SWE values are drawn toward the same observed coarse-scale value. The spatial variability in the analysis result is much smaller than in the forecast. The same holds for the 3D-Fm (not shown), but the analysis has slightly more spatial variability, because of the interaction between neighboring innovations at each point. With the 3D-C1, the cloud of finescale pixel values is moved toward the observation, based on the coarsescale difference between the observation and the forecast, but the forecasted spatial variability is generally maintained. The perhaps most interesting finding is that for the $3 \mathrm{D}-\mathrm{Cm}$, the spatial variability of the analysis is strongly improved and better mimics the truth, indicated by the analysis scatter points lined up around the 1-1 line. Apparently, including neighboring observations and a reasonable forecast error structure can help to improve the finescale SWE estimation over using only the observation at the analysis point of interest.

We also examined the spatial correlation coefficient between SWE assimilation analysis fields and the reference truth. The correlation coefficient measures the skill of the assimilation estimates in terms of finescale spatial structure. Figure 7 shows the time series mean of the correlation coefficient for each coarse-scale pixel area and for a variety of filter scenarios. By construction, the spatial correlation coefficient between the synthetic observations and the truth is negligible, because the observations are purely randomly perturbed. The filters with disaggregated observations (1D-F1 and 3D-Fm) degrade the spatial patterns with respect to the open loop. Overall, the 3D-C1 has only a limited positive effect and the spatial structure is only better than the open loop when 1_corr $>20-30 \mathrm{~km}$. However, the 3D-Cm scenarios with larger 1_corr show a great correlation improvement, mainly in the center coarse areas that benefit most from 



FIG. 6. Comparison of the (left) finescale forecasts and (right) analyses with the true SWE distributions within a single $25 \times 25 \mathrm{~km}^{2}$ coarse pixel (second row, second column of coarse grid) at 1 particular time step (30 Apr 2003; 0800 UTC). The dashed lines indicate the spatial means and the box shows the assimilated coarse-scale observation value.

surrounding information and a relatively gentle topography (and hence relatively isotropic precipitation error correlation field). For the coarse grid cell $(2,2)$, for example, the correlation coefficient increases from a mean of 0.15 for the open loop to 0.64 for the 3D-Cm with 1_corr $=20 \mathrm{~km}$. Only in two grid cells [i.e., $(3,2)$ and $(3,3)]$ does the $3 \mathrm{D}-\mathrm{Cm}$ not improve the spatial variability, which is most likely because of the simple forecast error correlation structure in combination with a highly dynamic and spatially variable snowpack distribution in that area.

Figure 5 shows the temporal SWE evolutions at two individual finescale points within the coarse region $(2,2)$. Most of the analyses better approach the truth than either the open loop or the observations, but none of the 1D and 3D filters are necessarily optimal at all locations within the coarse-scale pixel. At finescale grid cells where the disaggregated coarse observations are consistently biased when compared to the finescale truth, 1D-F1 assimilation will degrade the analysis results compared to the open loop [e.g., during the snow accumulation period in pixel $(49,49)]$. The 1D approach with disaggregated observation values has an observation bias problem at some individual locations. This problem can be overcome by assimilating the observations at the coarse-scale with a well-defined error correlation structure and the proper scaling observation operator (as in $3 \mathrm{D}-\mathrm{C} 1$ or $3 \mathrm{D}-\mathrm{Cm}$ ). When using multiple observations (i.e., 3D-Fm and $3 \mathrm{D}-\mathrm{Cm}$ ) for each update, the SWE analysis for a given finescale pixel can exceed (or be less than) the forecast and the local observation. This is mainly obvious during the melt phase, when some patches still have a high SWE value with a high forecast uncertainty, while other patches have a very shallow snowpack with a very limited uncertainty. An innovation at the deeper snowpack in the influence area around an analysis point with a limited SWE strongly impacts that analysis update (limited SWE means limited uncertainty and limited update by the 


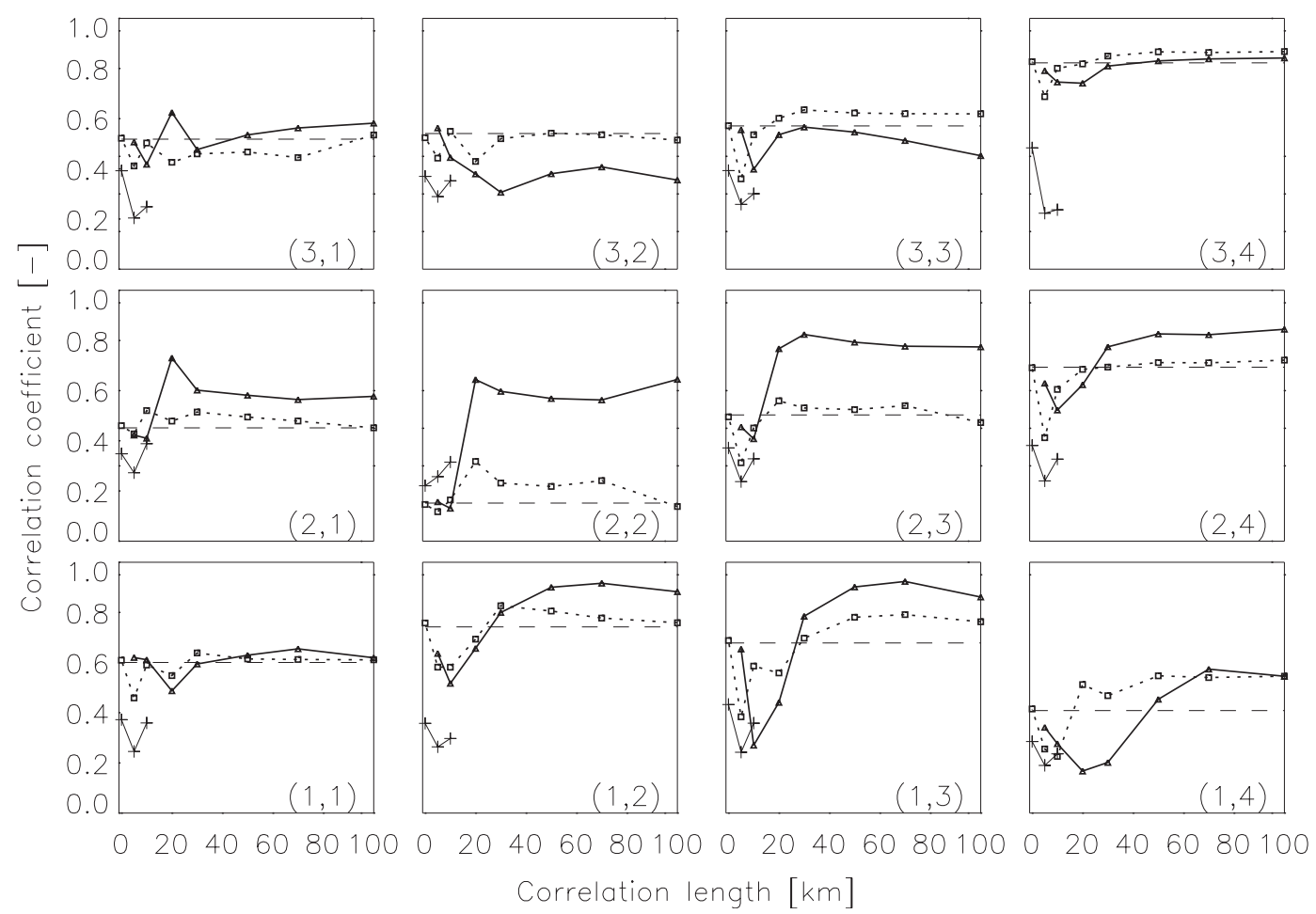

FIG. 7. Temporal mean of the spatial correlation coefficient between analyzed and true SWE as function of perturbations correlation length for (dashed) open loop, (black dotted) 3D-C1, and (black solid) 3D-Cm. Methods 1D-F1 (1_corr $=0 \mathrm{~km})$ and 3D-Fm (1_corr $=5$ and $10 \mathrm{~km})$ are shown with + symbols: (top left to bottom right) correlation length $(3,1)$ to $(1,4)$. Each subplot corresponds to a coarse grid cell (Fig. 1).

collocated observation). In general, the 3D-Cm analyses are closest to the true reference simulation. For 3D-C1, most analyses are moved toward the same direction with respect to the forecast, but that is not necessarily the best solution at each finescale location. The 3D-C1 filter with a small 1_corr results in an analysis close to the open loop, because of the small Kalman gain, as discussed earlier.

\section{5) Filter diagnostics}

Figure 8 shows the frequency distribution of the normalized ensemble mean innovations for different assimilation cases, that is, each innovation value $\left(\mathbf{y}_{i}-\mathbf{H}_{i} \hat{\mathbf{x}}_{i}^{-}\right)_{k}$ (at the fine scale) or $\left(\mathbf{y}_{i}-\mathbf{H}_{i} \hat{\mathbf{x}}_{i}^{-}\right)_{\kappa}$ (at the coarse scale) is normalized by the square root of its filter-estimated standard deviation $\left[\mathbf{H}_{i} \mathbf{P}_{i}^{-} \mathbf{H}_{i}^{\mathrm{T}}+\mathbf{R}_{i}\right]_{k k}^{1 / 2}$ or $\left[\mathbf{H}_{i} \mathbf{P}_{i}^{-} \mathbf{H}_{i}^{\mathrm{T}}+\mathbf{R}_{i}\right]_{\kappa \kappa}^{1 / 2}$ (fine or coarse scale, respectively). The normalized ensemble mean innovations should approximately obey a standard-normal distribution (Gaussian with mean zero and variance one), if the model is linear and the filter operates in accordance with its underlying assumptions. The innovation histograms provide a rough indication of whether the model and observation error parameters are appropriately chosen or not. The histograms show all innovations over space and time (for the 3D-C1 and
3D-Cm, there are only $12 \times 19$ innovations, while for the $1 \mathrm{D}-\mathrm{F} 1$ and $3 \mathrm{D}-\mathrm{Fm}$ there are $7500 \times 19)$. The figure shows a small bias in the innovation distributions. This is caused by local biases in the forcings. When a daily assimilation is performed during the period from 30 September 2002 to 30 June 2003 (274 assimilation time steps instead of 19), the histograms (not shown) exhibit similar features, but they show no bias because there is not enough forecast time to develop it.

For most filter scenarios, the standard deviation of the histograms is close to 1, indicating that the filter parameters are near optimal. During early snow accumulation and late ablation, the forecast uncertainty is bounded and it increases during deep snowpacks. Similarly, the observation error was increased with deeper snowpacks. This similar trend in observation and forecast errors keeps the filter close to its optimal operation during the entire snow season. However, with real observations, it remains to be seen how the observation error evolves in time and how it compares to the forecast uncertainty in each time period. Of all filter configurations, 3D-C1 with 1_corr $=0 \mathrm{~km}$ suggests the least optimal filter operation, with an excessive innovation spread. This is because the update is too limited to reduce the forecast uncertainty during 

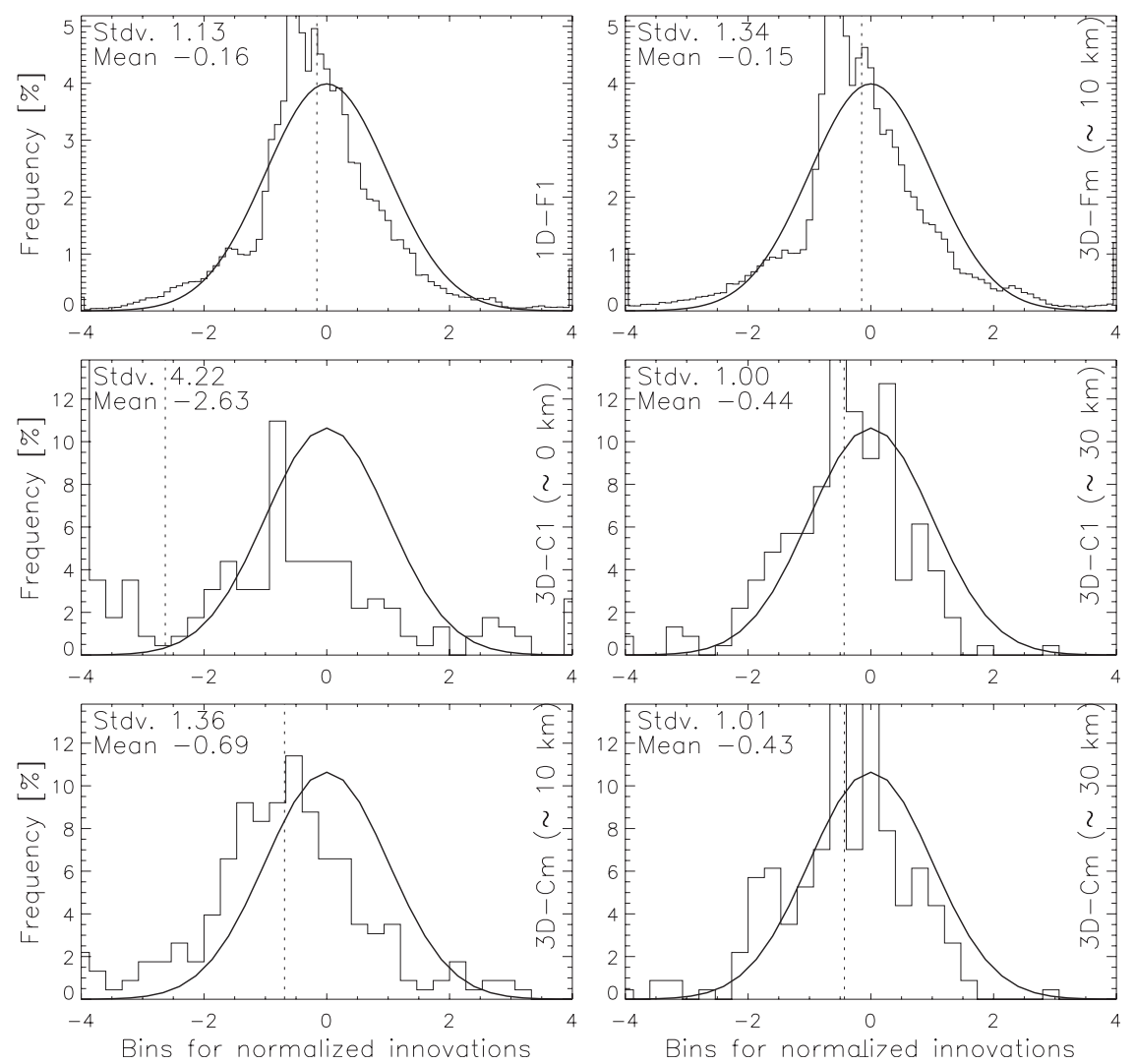

FIG. 8. Histograms of normalized innovations for different assimilation approaches.

filtering. Observation error variance sensitivity tests (not shown) indicate that modifying the observation error variance for the disaggregated observations could slightly reduce the RMSE for the 3D-Fm filter (for the specified forecast error). However, the corresponding innovation statistics show a suboptimal filter operation. This suggests that the better RMSE estimates may have resulted from compensation for imperfectly defined model errors.

Note that the innovation diagnostics are always available, including when real satellite observations are assimilated, and can be used to identify obviously inadequate assimilation parameters ( such as 1_corr $=0 \mathrm{~km}$ for $3 \mathrm{D}-\mathrm{C} 1)$ in the absence of reliable validation data.

\section{Conclusions}

The assimilation of coarse-scale $(25 \mathrm{~km})$ snow water equivalent (SWE) observations into finescale $(1 \mathrm{~km})$ model simulations is studied through a number of synthetic experiments with GMAO EnKF variants implemented in LIS5.0. Both a one-dimensional (1D) or point filter and a variety of three-dimensional (3D) or spatial filters are explored in a synthetical study. The truth is generated at the fine scale by forcing the Noah model with NLDAS data. Synthetic observations are generated through aggregation of these finescale simulations and addition of observation error. To assure a reasonable coefficient of variation in the observation error and in favor of the filter performance, the observation error variance is a function of the spatial mean SWE amounts. A degraded open loop model integration is simulated by forcing the Noah model with coarse GDAS data and imposing different types of spatially correlated random perturbations.

It is shown that coarse satellite products can improve both the SWE spatial mean and variability estimation over the open loop simulations, or in other words, finescale model simulations can downscale coarse satellite products to extract useful information for the finescale SWE estimation. Because of the temporal evolution of the SWE amounts over the winter, the forecast uncertainty is small during early accumulation and later during the melting season. Therefore, during the first days of simulated snow accumulation, the very small simulation spread and the fixed minimum observation error limits the assimilation impact. The month-long delay in snowmelt predicted by the open loop integration can be mostly removed through assimilation. 
When the coarse observations are disaggregated prior to assimilation, a number of problems arise. The 1D filter pushes the analyses to the coarse observation value, which improves the spatial mean SWE estimation but removes most of the subpixel spatial variability. Furthermore, significant finescale observation bias can be observed. If a number of finescale observations are used to update each analysis point in the 3D-Fm, the spatial variability is slightly better, but the computational cost becomes increasingly expensive in the inversion part of the Kalman gain calculation. For the actual assimilation of passive microwave products (e.g., AMSR-E), disaggregating the coarse observations to finer-scale observations may render the observations nearly useless, because the coarse products already have an unfavorable signal-to-noise ratio. The correct scaling of the observation error for disaggregated observations needs further research to optimize the filter at the fine scale.

The best approach is to assimilate the coarse-scale observations directly with a 3D filter and a properly defined forecast error correlation structure. For all 3D filters using the coarse observations, it is found that the analyses are best when the spatial forecast error correlation length is equal to or larger than $20-30 \mathrm{~km}$, which corresponds to the dimension of the coarse observation pixels, and also to the approximate correlation length of the precipitation error field. The results degrade significantly for shorter correlation lengths. When each finescale grid point is updated using the overlying coarse observation only (3D-C1), the spatial mean SWE field can be improved over that of the observations or open loop simulations alone, and the spatial subpixel variability can be enhanced slightly. With additional inclusion of surrounding coarse observations (3D-Cm), there is a significant improvement in estimating the subpixel variability. Furthermore, artificial boundaries in the analysis field, caused by the boundaries in the coarse observations, are completely removed.

Even though the entire study domain is observed, there is a substantial value in including coarse observations from neighboring areas to enhance the finescale SWE structure estimation within the $3 \mathrm{D}-\mathrm{Cm}$ filter. The SWE estimation can probably be further improved after optimization of the spatial error structure and covariance localization for the forecast error covariances in spatially complex terrain areas. In summary, from the four tested algorithms, the 3D-Cm filter is the method of choice to assimilate coarse observations, because it (i) improves the spatial mean SWE analysis, (ii) substantially enhances the subpixel SWE variability estimation, (iii) avoids artificial transitions at the coarse observation boundaries, and (iv) is computationally no more expensive than any of the other filter approaches.
As mentioned in section 2, passive microwave retrievals from current satellite sensors have limited sensitivity for deep snowpacks. Our results, which included assimilation of SWE retrievals beyond the saturation threshold (albeit with large errors) thus overestimate the improvements that can be expected from the assimilation and downscaling of current satellite SWE products, as remains to be demonstrated in future studies. With hindsight, a different study domain with less snow accumulation might have been preferable. Note, however, that the key results regarding the best method for the spatial disaggregation of satellite SWE estimates are not sensitive to this issue.

Acknowledgments. The first author is a postdoctoral research fellow of the Research Foundation Flanders (FWO). Rolf Reichle was supported by NASA Grant NNX08AH36G. We thank Sujay Kumar and the LIS development team at NASA GSFC for providing the LIS5.0 beta version, Hiroko Kato from NASA GSFC for providing the GDAS forcing data, and Megan Larko from CREW/IGES for the computational support. This study is partially supported by the NOAA CPPA Grant NA07OAR4310221. We thank the reviewers for their constructive comments.

\section{REFERENCES}

Andreadis, K. M., and D. P. Lettenmaier, 2006: Assimilating remotely sensed snow observation into a macroscale hydrology model. Adv. Water Resour., 29, 872-886.

Burgers, G., P. J. van Leeuwen, and G. Evensen, 1998: On the analysis scheme in the ensemble Kalman filter. Mon. Wea. Rev., 126, 1719-1728.

Caparrini, F., F. Castelli, and D. Entekhabi, 2004: Variational estimation of soil and vegetation turbulent transfer and heat flux parameters from sequences of multisensor imagery. Water Resour. Res., 40, W12515, doi:10.1029/2004WR003358.

Cosgrove, B. A., and Coauthors, 2003: Real-time and retrospective forcing in the North American Land Data Assimilation System (NLDAS) project. J. Geophys. Res., 108, 8842, doi:10.1029/ 2002JD003118.

Crow, W. T., and E. F. Wood, 2002: The value of coarse-scale soil moisture observations for regional surface energy balance modeling. J. Hydrometeor., 3, 467-482.

De Lannoy, G. J. M., R. H. Reichle, P. R. Houser, V. R. N. Pauwels, and N. E. C. Verhoest, 2007: Correcting for forecast bias in soil moisture assimilation with the ensemble Kalman filter. Water Resour. Res., 43, W09410, doi:10.1029/2006WR005449.

— , P. R. Houser, N. E. C. Verhoest, and V. R. N. Pauwels, 2009: Adaptive soil moisture profile filtering for horizontal information propagation in the independent column-based CLM2.0. J. Hydrometeor., 10, 766-779.

Derksen, C. A., A. Walker, E. LeDrew, and B. Goodison, 2003: Combining SMMR and SMM/I data for time series analysis of central North American snow water equivalent. J. Hydrometeor., 4, 304-316. 
Dirmeyer, P., 2000: Using a global soil wetness dataset to improve seasonal climate simulation. J. Climate, 13, 2900-2921.

Dong, J., J. P. Walker, and P. R. Houser, 2005: Factors affecting remotely sensed snow water equivalent uncertainty. Remote Sens. Environ., 97, 68-82.

,,--- , and C. Sun, 2007: Scanning multichannel microwave radiometer snow water equivalent assimilation. J. Geophys. Res., 112, D07108, doi:10.1029/2006JD007209.

Dubayah, R., E. F. Wood, and D. Lavalee, 1997: Multiscaling analysis in distributed modeling and remote sensing: An application using soil moisture. Scale, Multiscaling, Remote Sensing, and GIS, M. F. Goodchild and D. A. Quattrochi, Eds., Cambridge University Press, 93-111.

Durand, M., and S. A. Margulis, 2006: Feasibility test of multifrequency radiometric data assimilation to estimate snow water equivalent. J. Hydrometeor., 7, 443-457.

$\longrightarrow$, and — 2007: Correcting first-order errors in snow water equivalent estimates using a multifrequency, multiscale radiometric data assimilation scheme. J. Geophys. Res., 112, D13121, doi:10.1029/2006JD008067.

, and - 2008: Effects of uncertainty magnitude and accuracy on assimilation of multiscale measurements for snowpack characterization. J. Geophys. Res., 113, D02105, doi:10.1029/ 2007JD008662.

— N. Molotch, and S. A. Margulis, 2008: Merging complementary remote sensing datasets in the context of snow water equivalent. Remote Sens. Environ., 112, 1212-1225.

Ek, M., K. Mitchell, L. Yin, E. Rogers, P. Grunmann, V. Koren, G. Gayno, and J. D. Tarpley, 2003: Implementation of Noah land surface model advances in the National Centers for Environmental Prediction operational mesoscale Eta model. J. Geophys. Res., 108, 8851, doi:10.1029/2002JD003296.

Evensen, G., 2003: The ensemble Kalman filter: Theoretical formulation and practical implementation. Ocean Dyn., 53, 343-367.

Foster, J. L., C. Sun, J. P. Walker, R. Kelly, A. Chang, J. Dong, and H. Powell, 2005: Quantifying the uncertainty in passive microwave snow water equivalent observations. Remote Sens. Environ., 92, 187-203.

Gaspari, G., and S. Cohn, 1999: Construction of correlation functions in two and three dimensions. Quart. J. Roy. Meteor. Soc., 125, 723-757.

Goodison, B. E., 1978: Accuracy of Canadian snow gage measurements. J. Appl. Meteor., 17, 1542-1548.

Hamill, T. M., and C. Snyder, 2000: A hybrid ensemble Kalman filter-3D Variational analysis scheme. Mon. Wea. Rev., 128, 2905-2919.

Houser, P. R., W. J. Shuttleworth, J. S. Famiglietti, H. V. Gupta, K. H. Syed, and D. C. Goodrich, 1998: Integration of soil moisture remote sensing and hydrologic modeling using data assimilation. Water Resour. Res., 34, 3405-3420.

Huang, H.-C., and N. Cressie, 1996: Spatio-temporal prediction of snow water equivalent using the Kalman filter. Comput. Stat. Data Anal., 22, 159-175.

Hunt, B. R., E. J. Kostelich, and I. Szunyogh, 2006: Efficient data assimilation for spatiotemporal chaos: A local ensemble transform Kalman filter. Physica D, 230, 112-126.

Kelly, R. E., A. T. Chang, L. Tsang, and J. L. Foster, 2003: A prototype AMSR-E global snow area and snow depth algorithm. IEEE Trans. Geosci. Remote Sens., 41, 230-242.

Keppenne, L. C., and M. M. Rienecker, 2002: Initial testing of a massively parallel ensemble Kalman filter with the Poseidon Isopycnal Ocean General Circulation model. Mon. Wea. Rev., 130, 2951-2965.
Koster, R. D., and Coauthors, 2004: Realistic initialization of land surface states: Impacts on subseasonal forecast skill. $J$. Hydrometeor., 5, 1049-1063.

Kumar, P., 1999: A multiple scale state-space model of characterizing subgrid scale variability of near-surface soil moisture. IEEE Trans. Geosci. Remote Sens., 37, 182-197.

Kumar, S. V., and Coauthors, 2006: Land Information System: An interoperable framework for high resolution land surface modeling. Environ. Model. Software, 21, 1402-1415. , C. D. Peters-Lidard, J. L. Eastman, and W.-K. Kao, 2008a: An integrated high-resolution hydrometeorological modeling test bed using LIS and WRF. Environ. Model. Software, 23, 169-181.

—, R. H. Reichle, C. D. Peters-Lidard, R. D. Koster, X. Zhan, W. T. Crow, J. B. Eylander, and P. R. Houser, 2008b: A land surface data assimilation framework using the Land Information System: Description and applications. Adv. Water Resour., 31, 1419-1432.

Liang, T., X. Zhang, H. Xie, C. Wu, Q. Feng, X. Huang, and Q. Chen, 2008: Toward improved daily snow cover mapping with advanced combination of MODIS and AMSR-E measurements. Remote Sens. Environ., 112, 3750-3761.

Liston, G., and C. A. Hiemstra, 2008: A simple data assimilation system for complex snow distributions (SnowAssim). J. Hydrometeor., 9, 989-1004.

$\longrightarrow$, R. Pielke, and E. Greene, 1999: Improving first-order snowrelated deficiencies in a regional cimate model. J. Geophys. Res., 104 (D16), 19 559-19 567.

Merlin, O., A. Chehbouni, G. Boulet, and Y. Kerr, 2006: Assimilation of disaggregated microwave soil moisture into a hydrologic model using coarse-scale meteorological data. J. Hydrometeor., 7, 1308-1322.

Mitchell, K. E., and Coauthors, 2004: The multi-institution North American Land Data Assimilation System (NLDAS): Utilizing multiple GCIP products and partners in a continental distributed hydrological modeling system. J. Geophys. Res., 109, D07S90, doi:10.1029/2003JD003823.

Mote, T. L., A. J. Grundstein, D. J. Leathers, and D. A. Robinson, 2003: A comparison of modeled, remotely sensed, and measured snow water equivalent in the northern Great Plains. Water Resour. Res., 39, 1209, doi:10.1029/2002WR001782.

Ott, E., and Coauthors, 2004: A local ensemble Kalman filter for atmospheric data assimilation. Tellus, 56A, 415-428.

Pan, M., and E. F. Wood, 2006: Data assimilation for estimating the terrestrial water budget using a constrained ensemble Kalman filter. J. Hydrometeor., 7, 534-547.

,-- D. B. McLaughlin, D. Entekhabi, and L. Luo, 2009: A multiscale ensemble filtering system for hydrologic data assimilation. Part I: Implementation and synthetic experiment. J. Hydrometeor., 10, 794-806.

Parada, L. M., and X. Liang, 2003: A downscaling framework for L band radio brightness temperature imagery. J. Geophys. Res., 108, 8863, doi:10.1029/2003JD003637.

— , and — 2004: Optimal multiscale Kalman filter for assimilation of near-surface soil moisture into land surface models J. Geophys. Res., 109, D24109, doi:10.1029/2004JD004745.

$\ldots$, and _ 2008: Impacts of spatial resolutions and data quality on soil moisture data assimilation. J. Geophys. Res., 113, D10101, doi:10.1029/2007JD009037.

Pauwels, V. R. N., and G. J. M. De Lannoy, 2006: Improvement of modeled soil wetness conditions and turbulent fluxes through the assimilation of observed discharge. J. Hydrometeor., 7, $458-477$. 
Peters-Lidard, C. D., and Coauthors, 2007: High-performance earth system modeling with NASA/GSFC's Land Information System. Innovat. Syst. Software Eng., 3, 157-165.

Pulliainen, J., 2006: Mapping of snow water equivalent and snow depth in boreal and sub-arctic zones by assimilating spaceborne microwave radiometer data and ground-based observations. Remote Sens. Environ., 101, 257-269.

__ J. Grandell, and M. Hallikainen, 1999: HUT snow emission model and its applicability for snow water equivalent retrieval. IEEE Trans. Geosci. Remote Sens., 37, 1378-1390.

Reichle, R. H., and R. Koster, 2003: Assessing the impact of horizontal error correlations in background fields on soil moisture estimation. J. Hydrometeor., 4, 1229-1242.

$\longrightarrow$, and - 2004: Bias reduction in short records of satellite soil moisture. Geophys. Res. Lett., 31, L19501, doi:10.1029/ 2004GL020938.

- and - 2005: Global assimilation of satellite surface soil moisture retrievals into the NASA Catchment land surface model. Geophys. Res. Lett., 32, L02404, doi:10.1029/ 2004GL021700.

_ D. Dntekhabi, and D. B. McLaughlin, 2001: Downscaling of radio brightness measurements for soil moisture estimation: A four dimensional variational data assimilation approach. Water Resour. Res., 37, 2353-2364.

— D. B. McLaughlin, and D. Entekhabi, 2002a: Hydrologic data assimilation with the ensemble Kalman filter. Mon. Wea. Rev., 130, 103-114.

— , J. P. Walker, P. R. Houser, and R. D. Koster, 2002b: Extended versus ensemble Kalman filtering for land data assimilation. J. Hydrometeor., 3, 728-740.

— , R. Koster, P. Liu, S. P. P. Mahanama, E. G. Njoku, and M. Owe, 2007: Comparison and assimilation of global soil moisture retrievals from the Advanced Microwave Scanning Radiometer for the Earth Observing System (AMSR-E) and the Scanning Multichannel Microwave Radiometer (SMMR). J. Geophys. Res., 112, D09108, doi:10.1029/2006JD008033.

, M. G. Bosilovich, W. T. Crow, R. D. Koster, S. V. Kumar, S. P. P. Mahanama, and B. F. Zaitchik, 2009: Recent advances in land data assimilation at the NASA Global Modeling and Assimilation Office. Data Assimilation for Atmospheric, Oceanic, and Hydrologic Applications, S. K. Park and L. Xu, Eds., Springer Verlag, 407-428.

Rodell, M., and P. R. Houser, 2004: Updating a land surface model with MODIS-derived snow cover. J. Hydrometeor., 5, 1064-1075.

Slater, A. G., and M. Clark, 2006: Snow data assimilation via an ensemble Kalman filter. J. Hydrometeor., 7, 478-493.

_ and Coauthors, 2001: The representation of snow in land surface schemes: Results from PILPS 2(d). J. Hydrometeor., 2, 7-25.

Sun, C., J. P. Walker, and P. R. Houser, 2004: A methodology for snow data assimilation in a land surface model. J. Geophys. Res., 109, D08108, doi:10.1029/2003JD003765.

Tait, A. B., D. K. Hall, J. L. Foster, and R. L. Armstrong, 2000: Utilizing multiple datasets for snow-cover mapping. Remote Sens. Environ., 72, 111-126.

Zaitchik, B. F., and M. Rodell, 2009: Forward-looking assimilation of MODIS-derived snow-covered area into a land surface model. J. Hydrometeor., 10, 130-148.

, and R. H. Reichle, 2008: Assimilation of GRACE terrestrial water storage data into a land surface model: Results for the Mississippi River basin. J. Hydrometeor., 9, 535-548.

Zhou, Y., D. McLaughlin, D. Entekhabi, and G. Crystal Ng, 2008: An ensemble multiscale filter for large nonlinear data assimilation problems. Mon. Wea. Rev., 136, 678-698. 